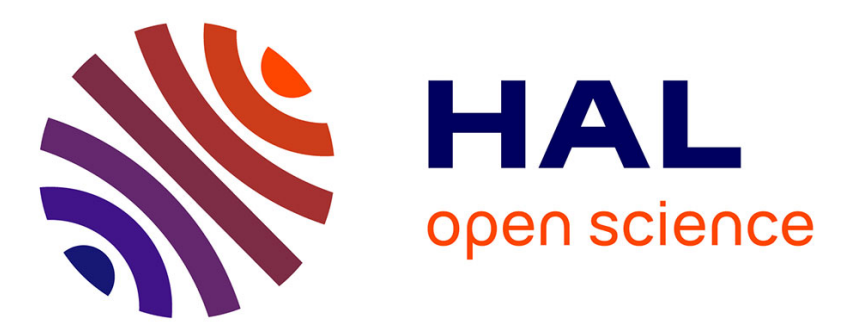

\title{
Cahier de formation continue Information et vécu parental du diagnostic de l'autisme II. Effets des troubles et qualité de vie
}

L. Beaud, Jean-Claude Quentel

\section{- To cite this version:}

L. Beaud, Jean-Claude Quentel. Cahier de formation continue Information et vécu parental du diagnostic de l'autisme II. Effets des troubles et qualité de vie. Annales Médico-Psychologiques, Revue Psychiatrique, 2011, 10.1016/j.amp.2010.12.010 . hal-00727596

\section{HAL Id: hal-00727596 \\ https://hal.science/hal-00727596}

Submitted on 4 Sep 2012

HAL is a multi-disciplinary open access archive for the deposit and dissemination of scientific research documents, whether they are published or not. The documents may come from teaching and research institutions in France or abroad, or from public or private research centers.
L'archive ouverte pluridisciplinaire HAL, est destinée au dépôt et à la diffusion de documents scientifiques de niveau recherche, publiés ou non, émanant des établissements d'enseignement et de recherche français ou étrangers, des laboratoires publics ou privés. 


\section{Accepted Manuscript}

Title: Cahier de formation continue Information et vécu parental du diagnostic de l'autisme II. Effets des troubles et qualité de vie

Authors: L. Beaud, J.-C. Quentel

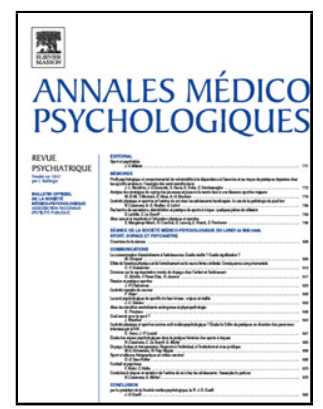

PII:

S0003-4487(10)00416-6

DOI: doi:10.1016/j.amp.2010.12.010

Reference: AMEPSY 1284

To appear in: Annales Médico-Psychologiques

Please cite this article as: Beaud L, Quentel J-C, Cahier de formation continue Information et vécu parental du diagnostic de l'autisme II. Effets des troubles et qualité de vie, Annales medio-psychologiques (2010), doi:10.1016/j.amp.2010.12.010

This is a PDF file of an unedited manuscript that has been accepted for publication. As a service to our customers we are providing this early version of the manuscript. The manuscript will undergo copyediting, typesetting, and review of the resulting proof before it is published in its final form. Please note that during the production process errors may be discovered which could affect the content, and all legal disclaimers that apply to the journal pertain. 
Cahier de formation continue

Information et vécu parental du diagnostic de l'autisme

II. Effets des troubles et qualité de vie

Parental experiences of autism and diagnosis issue

I. Effects of disorders and quality of life

L. Beaud ${ }^{\mathrm{a}, *}$, J.-C. Quentel ${ }^{\mathrm{a}}$

${ }^{a}$ Département Sociologie - Langage, Université Européenne de Bretagne, Place du recteur Henri Le Moal, CS 24307, 35043 Rennes cedex, France

Auteur correspondant : L. Beaud, Département Sociologie - Langage, Université européenne de Bretagne, Place du recteur Henri Le Moal, CS 24307, 35043 Rennes cedex, France

Adresse e-mail : laurence.beaud@univ-rennes2.fr

Résumé

Cet article constitue la deuxième partie d'un dossier qui a pour objectif de proposer un état des lieux de l'apport du vécu parental dans la compréhension diagnostique du « spectre autistique ». Le premier travail faisait le point sur les moments à la fois du repérage des premiers symptômes par les parents et du premier diagnostic, ainsi que sur la nature de leurs premières inquiétudes. Celui-la tente de cerner le retentissement psychosocial des troubles sur les parents, et donc plus généralement sur leur qualité de vie, ainsi que les stratégies qu'ils emploient pour faire face aux symptômes présentés par l'enfant. Le propos s'attache également à analyser les facteurs qui déterminent les différences observées. Notre hypothèse est que la mesure de ces effets participe également, en creux, de la connaissance du syndrome autistique et de son diagnostic.

Mots clés : Affects ; Autisme ; Diagnostic ; Parent ; Qualité de vie

Abstract

This article is part two of a study whose goal is to provide the current state of knowledge concerning the parental contribution in early identification and diagnostic patterns of children with autism spectrum disorder. The first article was an update on the age of recognition of early symptoms by parents and the initial diagnosis as well as the nature of concerns. The purpose of this one is to evaluate the psychosocial repercussions of child's trouble on parents, and therefore on their quality of life and their strategies used to cope with the disorder. Our goal is also to analyze the factors that determine the differences. We assume that the knowledge of these effects is also involved in the knowledge and diagnosis of the autistic spectrum disorder.

Keywords: Affects; Autism; Diagnosis; Parent; Quality of life

\section{Introduction}

Le retentissement psychosocial du syndrome autistique, ou d'un trouble envahissant du développement (TED), sur les familles, ainsi que leurs stratégies pour y faire face font l'objet d'un nouvel intérêt grandissant. Il s'agit dans le premier cas de mesurer l'épreuve affective que constituent les troubles, mais aussi leurs effets sur la vie conjugale, l'économie 
familiale, la fratrie, la santé physique et psychique de l'entourage [6,28,32,33]. Ces études répondent en fin de compte, mais à contresens, aux études antérieures qui cherchaient à mesurer l'impact éventuel des parents (de leurs caractéristiques psychopathologiques) sur l'autisme de leurs enfants, dans une perspective surtout étiologique. La tendance actuelle, diamétralement opposée, est la possibilité offerte aux professionnels de tirer parti des savoirs d'expérience parentaux pour, entre autres, mieux comprendre l'autisme [26]. C'est pourquoi nous allons retenir ici les études, recensées pour la plupart à partir de bases de données informatisées et de mots clés, qui suggèrent que le retentissement psychosocial serait différent selon l'identité du trouble dont l'enfant souffre. Les travaux mentionnant explicitement le vécu des parents ont été privilégiés. Peut-on alors comprendre le trouble à partir de ses effets sur les parents? Existe-t-il ou non un écart entre les premières inquiétudes ou préoccupations déclarées et leurs retentissements déclarés sur la qualité de vie ? Qu'est-ce qui est le plus éprouvant pour les parents? Les réponses à ces questions ne sont pas évidentes car le vécu qualitatif des parents est forcément filtré par des déterminismes de nature différente comme les caractéristiques de l'enfant autiste, la composition de la cellule familiale, l'accès aux services professionnels, ou encore le type de prise en charge.

En ce sens, les récits de vie mériteraient d'ailleurs une attention particulière. Il existe un certain nombre d'autobiographies de parents d'autistes ou de personnes autistes dont certaines sont coécrites. Moi, l'enfant autiste [3] est explicitement un livre écrit à deux voix : celle de Sean et de sa mère Judy. Une autre mère, Hilde De Clercq [8] témoigne notamment de l'hypersélectivité (ou de la «pensée en détail ») de son enfant autiste (Thomas) que l'on retrouve chez de nombreux Asperger. Le témoignage d'un père [25] est suffisamment rare, mais aussi détaillé dans sa description, pour être aussi mentionné. L'analyse narratologique de l'autisme reste peu répandue car elle pose des problèmes tant méthodologiques qu'épistémologiques (mentionnés dans notre article précédent). Mais l'intérêt du récit est de récapituler des événements qui apparaissent fragmentés, notamment pour les professionnels. Les récits de vie montrent d'ailleurs des similitudes dans la trajectoire de parents qui ne se présentent pas comme des victimes mais témoignent de leur volonté de faire face [9].

\section{L'épreuve parentale}

Le terme désigne les sentiments éprouvés par les parents, qu'ils soient négatifs, positifs, ou les deux à la fois. L'intérêt de l'étude de Hahaut et al. [13] est de donner la parole à 16 parents d'enfants âgés de 4 à 17 ans (moyenne d'âge de 9,4) ayant un trouble autistique 
«non spécifié » et qui fréquentent des classes d'enseignement spécialisé. Sans surprise, ce sont les «problèmes éducatifs » qui apparaissent les plus éprouvants affectivement pour tous les parents interrogés, en particulier l'apprentissage de la propreté $(n=10)$ et la gestion des comportements en public $(\mathrm{n}=10)$. En revanche, la gestion des colères ou de l'anxiété semble peu problématique. Ce résultat peut s'expliquer en partie par la familiarité ou l'adaptation des familles : elles connaissent et surtout évitent dans la mesure du possible les situations qui peuvent déstabiliser l'enfant. En revanche, le regard des autres et la stigmatisation sociale (l'enfant est souvent considéré par ceux qui ne le connaissent pas comme étant mal élevé) ont une influence importante sur l'épreuve qu'elles traversent, de même que le manque de réciprocité émotionnelle de l'enfant. Les auteurs constatent également que les parents gardent du plaisir à jouer avec l'enfant et que dans la plupart des cas les relations entre l'enfant et la famille ou des amis ne sont pas sources de stress supplémentaire. Des observations en partie similaires ont été faites par Green [12] qui a interrogé 81 mères (États-Unis) d'enfants malades (paralysie cérébrale, syndrome de Down, autisme, spina bifida...). Pour la majorité des mères, la charge du soin (burden of care) est une question de contraintes essentiellement sociales (charge financière, de temps, impact sur le travail, la famille, les relations sociales, etc.), plutôt que de détresse émotionnelle ou de «charge subjective » (culpabilité, inquiétude, ressentiment, etc.). Ce résultat rejoint le constat selon lequel les thérapeutes ont tendance à dire que les familles sont plus stressées par les symptômes de leurs enfants qu'elles ne le disent elles-mêmes [4]. Malgré ces contraintes sociales liées à la garde d'un enfant ayant des besoins complexes, la plupart des mères (et surtout les mères de jeunes enfants) en retirent des «bénéfices» (par exemple amour, joie, fierté, courage; l'enfant rend meilleur, plus compétent, engage à regarder autrui au-delà des apparences...); en revanche, le stigmate social a un impact sur les deux dimensions de la charge et, dans son impact sur la charge subjective, peut diminuer les «bénéfices » perçus. Ce stigmate sous-tend en effet une critique de la compétence parentale. Dans l'étude de Meirsschaut et al. [19], les sentiments d'inefficacité, de culpabilité, de mésestime de soi ou d'impuissance sont plus fréquemment exprimés par les mères $(\mathrm{n}=17)$ ayant un enfant autiste (de 46 à 84 mois). Les difficultés maternelles à attirer l'attention de l'enfant, à l'amuser et à lui donner les soins corporels (nourrir, changer, baigner) apparaissent particulièrement distinctives par rapport à un enfant au développement typique.

L'étude de Myers et al. [21], convergente avec d'autres, est remarquable par sa précision en ce domaine. Quatre cent quatre-vingt-treize parents d'enfants ayant un TED (autisme, syndrome d'Asperger et «TED non spécifié ») ont répondu à un questionnaire (en 
ligne) sur la façon dont le syndrome autistique a affecté leur vie conjugale et familiale. Les participants sont à 92,2 \% des mères (âgées de 22 à 72 ans), mariées (79,6 \%); 9,2\% sont divorcées et $9 \%$ séparées, célibataires ou veuves. Les enfants ( $80 \%$ de garçons) sont âgés de 8,6 ans en moyenne. Cinq thèmes ressortent particulièrement des réponses par ordre de fréquence :

1) le stress ;

2) le comportement de l'enfant ;

3) la santé, le travail, la relation maritale ;

4) l'impact sur l'ensemble de la famille ;

5) l'isolement social.

Les réponses se répartissent de la façon suivante : impact positif ou négatif (48\% des réponses), mixte (39\% ; les parents mentionnent à la fois des effets négatifs : difficultés financières, faire avec les obsessions de leur enfant, dangers encourus... et positifs : une foi plus profonde, une plus grande patience...), et «ne sais pas » (ni positif ni négatif). Quarantesix parents (un sur dix) donnent une réponse complètement positive. Plus de $70 \%$ des parents expriment le stress, de façon explicite ou en évoquant les tensions conjugales, les soins et surveillance constants, les problèmes scolaires, les perturbations de la vie familiale, l'exclusion sociale... Ils « vivent un cauchemar », leur enfant « est un fardeau ».

Tableau 1 : analyse thématique des réponses des parents $(\mathrm{N}=493)$ à la question : «Comment l'autisme de votre enfant a-t-il affecté votre vie conjugale et familiale?» ([21, p. 675]; traduction personnelle)

\begin{tabular}{|l|c|}
\hline & $\mathrm{N}$ \\
\hline CLUSTER 1 - Stress & \\
\hline Thèmes négatifs : & 350 \\
\hline (pas de thèmes positifs) & \\
\hline CLUSTER 2 - Comportement de l'enfant et prises en charge & \\
\hline Thèmes négatifs : & 48 \\
\hline Difficultés à s'occuper des problèmes de comportement de l'enfant & 45 \\
\hline Accaparement de temps et dépense d'énergie pour les soins et les thérapies & 31 \\
\hline Problèmes de sommeil, épuisement & 28 \\
\hline Lutter avec les écoles publiques et les services administratifs & \\
\hline Thèmes positifs : & 19 \\
\hline Nouvelle compréhension et sensibilité par rapport au monde du handicap (incluant l'engagement militant) & 12 \\
\hline $\begin{array}{l}\text { L'autisme de l'enfant est une expérience exceptionnelle, unique, intéressante ; « on l'aime comme il est », «il } \\
\text { procure de la joie, de l'humour... » }\end{array}$ & \\
\hline CLUSTER 3 - Influence sur le bien-être des parents et la relation conjugale & \\
\hline Thèmes négatifs : & 75 \\
\hline Tension conjugale/maritale (incluant divorce et séparation) ; pas de vie intime & 44 \\
\hline Affects négatifs : peine, dépression, culpabilité, reproche... & 36 \\
\hline $\begin{array}{l}\text { Carrière professionnelle de la mère ou du père affectée négativement (abandon du travail, ou nécessité de plusieurs } \\
\text { emplois...) }\end{array}$ & \\
\hline Thèmes positifs : & \\
\hline
\end{tabular}




\begin{tabular}{|c|c|}
\hline Enrichissement, bénédiction, amour de l'enfant en dépit des difficultés & 50 \\
\hline Affects positifs : apprentissage de la compassion, tolérance, la patience, la joie... & 42 \\
\hline Apprendre à apprécier des petites choses, apprécier chaque moment, prendre le temps de vivre... & 39 \\
\hline Une vie spirituelle enrichie & 16 \\
\hline Mariage enrichi, renforcé & 8 \\
\hline \multicolumn{2}{|l|}{ CLUSTER 4 - Influence sur la famille entière (fratrie et famille étendue) } \\
\hline \multicolumn{2}{|l|}{ Thèmes négatifs : } \\
\hline Fratrie négligée, embarrassée ou agressée & 73 \\
\hline Tension financière (coût des thérapies, des écoles spécialisées, des régimes alimentaires, des assurances...) & 48 \\
\hline L'autisme est au centre de notre vie, et a tout changé, pas de vie à soi & 46 \\
\hline Tensions dans les relations avec la famille étendue & 16 \\
\hline Thèmes positifs : & \\
\hline Ajustement et aide familiaux positifs, rend la famille plus forte et unie & 21 \\
\hline Impact positif sur la fratrie (compassion, tolérance...) & 18 \\
\hline \multicolumn{2}{|l|}{ CLUSTER 5 - Isolement social } \\
\hline \multicolumn{2}{|l|}{ Thèmes négatifs : } \\
\hline Limitation des lieux où aller et de ce que l'on peut faire (pas de vacances, de visites, ne pas aller au restaurant...) & 67 \\
\hline Perte d'amis, pas de vie sociale & 43 \\
\hline Mauvais traitements, commentaires déplaisants des étrangers ou autres & 11 \\
\hline (pas de thèmes positifs) & \\
\hline
\end{tabular}

On mesure ici à quel point le syndrome autistique se dessine en creux dans les effets (négatifs) le plus souvent cités : à l'isolement social de l'enfant, spécifique du trouble autistique (aloneness), répond l'isolement familial. Au trouble de l'intimité (de la familiarité, du « contact affectif ») répondent des perturbations de l'intimité conjugale ou familiale. On remarquera aussi l'attitude malgré tout positive d'un grand nombre de parents (cf. cluster 3). Il reste que l'absence, même partielle, de réciprocité dans les interactions verbales ou non verbales apparaît particulièrement éprouvante. L'étude de Osborne et Reed [23] montre à quel point le sentiment d'une moindre implication parentale et une communication pauvre avec l'enfant autiste (de moins de 4 ans surtout) sont des facteurs de stress pour les parents ( $\mathrm{n}=138$; enfants de 2 à 16 ans).

\section{Les stratégies parentales}

Certaines études s'intéressent plus particulièrement aux stratégies du «faire face » (coping) mises en place par les parents. Celle de Pottie et al. [27], par exemple, analyse le stress et l'humeur, ainsi que les façons d'y répondre, de 93 parents (64 \% de mères/36 \% de pères) d'enfants de 7,3 ans (63 autistes, 27 TED non spécifié ou PDD-NOS, trois Asperger). Les parents devaient répondre, deux fois par semaine sur une période de 12 semaines, à la question suivante : "Quel a été le problème le plus exigeant, difficile ou gênant de la journée ? Décrivez-le brièvement ». Ils devaient ensuite dire, parmi les 11 stratégies suivantes, laquelle avait été utilisée :

1) recherche de soutien ; 


\section{2) échappatoire ;}

3) essayer de résoudre le problème ;

4) chercher des distractions ;

5) faire des reproches ou diriger sa colère contre quelqu'un ;

6) positiver ;

7) être négatif ;

8) contrôler sa détresse ;

9) s’isoler ou éviter ;

10) faire des compromis (entre les priorités/besoins de l'enfant et le coût émotionnel) ;

11) se sentir démuni ou dépassé.

Ils devaient choisir également parmi 20 adjectifs (par exemple soucieuse, forte, nerveuse, affligée...) ceux qui leur apparaissaient les plus descriptifs de leur humeur du jour. Les résultats sont peu détaillés mais cinq stratégies $(1,3,6,8,10)$ apparaissent prédictives d'une humeur plus positive, tandis que quatre $(2,5,9,11)$ sont associées à une humeur négative. Le plus intéressant est de noter que le genre n'a pas d'effet modulateur sur ces effets, mais trois stratégies (régulation émotionnelle, soutien social et inquiétudes) semblent avoir un effet de mesure sur le stress ou la qualité des relations avec l'enfant. Ni la symptomatologie autistique (mesurée par The Social Communication Questionnaire-Lifetime Form, autoquestionnaire basé sur le DSM-IV), ni la distance d'avec le diagnostic ne semblent affecter l'humeur parentale (même si le temps écoulé semble toutefois diminuer le stress). Benson [5] a étudié les méthodes de 113 mères d'enfants autistes (8,6 ans de moyenne d'âge). Les stratégies d'évitement (recherche de distractions par le divertissement, le travail, l'humour...) et de désengagement (usage de drogue, désengagement comportemental, déni) sont associées à une augmentation de la dépression et de la colère maternelle, alors que la stratégie du recadrage ou de redéfinition (acceptation, religion, positivisme...) est associée à un niveau de bien-être élevé. Plus la mère déclare jouer un rôle actif au quotidien (par exemple jouer longtemps avec son enfant) et dans le développement de son enfant, et plus le sentiment de compétence augmente; à l'inverse, plus la mère rapporte des sentiments dépressifs, de culpabilité, et plus le niveau de compétence est bas [16] (sur un échantillon de 170 mères d'enfants de 6,5 ans en moyenne).

Parmi les souffrances des parents, celle de ne pas comprendre son enfant est tout aussi éprouvante. Mais les parents cherchent toujours à donner du sens à leurs expériences et à expliquer en particulier les événements ou circonstances défavorables auxquels ils doivent 
faire face. En interrogeant 59 parents d'enfants Asperger (moyenne d'âge de 11 ans), Samios et al. [30] distinguent six types d'explication : une croyance spirituelle (c'est le destin, la main de Dieu...), l'attribution d'une origine à la pathologie (un traumatisme à la naissance...), un changement de perspective (j'ai appris beaucoup de choses...), l'identification (mon enfant est comme moi...), une redéfinition (ce n'est pas une pathologie mais une différence, qui s'estompera...), et enfin la chance/malchance. Ces stratégies se retrouvent dans l'étude de Veyssières et al. [34], qui analyse les difficultés passées (avant, au moment du diagnostic et juste après) et actuelles (au moment de l'étude) de 14 familles (guadeloupéennes) d'adolescents de 16 ans en moyenne, souffrant d'un autisme sévère (diagnostiqué entre 1980 et 1990, en France métropolitaine pour la moitié d'entre eux, et pris en charge en externat dans une IME; trois d'entre eux ont accès à un langage oral stéréotypé). Les auteurs distinguent trois stratégies de «faire face » à la situation juste après le diagnostic : le recours à la religion, le fatalisme qui aboutit à la résignation (le parent se «fait une raison»), et la réévaluation positive du problème (en soulignant ce que l'enfant a apporté ou appris aux parents).

\section{Les facteurs de différenciation}

\subsection{Selon le type de TED ou de pathologie}

La littérature suggère que la qualité de vie est différente selon la nature du trouble [32]. Le type de diagnostic peut donc avoir des impacts différents, en particulier sur le «stress » des parents. Les effets apparaissent nettement plus négatifs dans le cas de l'autisme que dans d'autres types de troubles (trisomie 21, paralysie cérébrale, $\mathrm{X}$ fragile...). Les parents d'enfants autistes ressentent plus de stress, sont plus déprimés et souffrent davantage que les parents d'enfants ayant d'autres types de handicaps. C'est ce qui ressort de l'étude de Roeyers et al. [29]. Les auteurs ont interrogé 80 familles belges (31 ont un enfant souffrant d'autisme bénéficiant d'une éducation spécialisée; 22 une déficience mentale; 31 un enfant non handicapé). Si le stress ou les symptômes dépressifs sont plus fréquents chez les parents ayant un enfant handicapé que chez les parents ayant un enfant non handicapé, ils sont aussi plus fréquents avec un enfant autiste qu'avec un enfant souffrant d'une déficience mentale. L'étude à grande échelle de Shieve et al. [31] aboutit au même résultat. Les parents d'enfants autistes ( $\mathrm{n}=459$, de 4 à 17 ans) sont beaucoup plus stressés, plus démunis et moins heureux que les parents d'enfants ayant un trouble de développement $(n=4545)$ autre que l'autisme, 
ou que les parents d'enfants nécessitant un soin (sans problèmes développementaux; $\mathrm{n}=11475)$, et que les parents d'enfants sans problèmes spéciaux $(11 \% ; \mathrm{n}=61826)$. Abbeduto et al. [1] comparent des enfants (de 10 à 23 ans) avec un syndrome de $\mathrm{X}$ fragile $(n=22)$, un syndrome de down $(n=39)$ et un syndrome autistique $(n=174)$. Les mères des premiers montrent un niveau de bien-être inférieur aux mères des seconds (moins de pessimisme...), mais supérieur aux mères d'enfants et adolescents autistes (qui montrent un plus haut niveau de pessimisme, une plus grande distance relationnelle, et un niveau de symptômes dépressifs plus élevé). Mugno et al. [20] comparent la qualité de vie de parents $(\mathrm{n}=212)$ d'enfants ou adolescents $(\mathrm{n}=135)$ atteints de TED (autisme typique, autisme de haut niveau ou asperger et autisme non spécifié), de paralysie cérébrale, et de retard mental, comparés à un groupe contrôle (77 parents et 48 enfants). Les problèmes de santé physique, psychologiques, sociaux, sont plus souvent rapportés par les parents d'enfants ayant un TED que dans les deux autres groupes. En revanche, il n'y a pas de différence significative entre les types de TED. Les parents d'enfants Asperger semblent cependant montrer une qualité de vie moindre que le groupe des autistes typiques.

Certaines caractéristiques de l'autisme, par rapport à d'autres pathologies, pourraient expliquer ces différences. Rappelons d'abord que le syndrome autistique est un trouble de la communication verbale et non verbale, de nature foncièrement sociale, mettant à l'épreuve directement la relation parent/enfant. Nous pouvons mentionner également des éléments aggravants: la rareté du trouble (impliquant un processus diagnostic long, compliqué et incertain); des comportements autistiques très difficiles à gérer (crise, obsession et rigidités de toutes sortes, et parfois automutilations); une compréhension et une tolérance publique peut-être plus faible (impliquant l'isolement familial); des traitements et des interventions éducatives coûteuses en temps, qui exigent une patience et une énergie au long cours, sans résultat garantis, de la part de l'entourage ; un avenir de l'enfant incertain. Il semblerait que l'incertitude concernant la cause et le pronostic de l'autisme pèse particulièrement sur la conception des mères. Nous pouvons facilement concevoir que plus le handicap pèse sur le devenir de l'enfant et plus le stress éprouvé par le parent est grand [7].

\subsection{Changements de l'adaptation parentale}

L'endurance des parents est également mise à mal. Gray [10] montre que la nature des difficultés rencontrées par les parents $(n=28 ; 19$ mères et 9 pères d'enfants âgés de 6 à 19 ans) ainsi que leurs stratégies changent dans le temps (après 8/10 ans). Ces difficultés sont 
particulièrement fortes au moment du diagnostic et focalisées spécifiquement sur les manques perçus chez l'enfant. Au fil du temps, l'utilisation des services diminue, de même que le soutien familial. Les parents se sentent en fait moins isolés socialement et les stratégies « individualistes » (travail, occupations...) diminuent, tandis que les stratégies de «faire face » (prendre la vie comme elle vient, contacter d'autres parents...) augmentent. Ces différences s'expliquent par le changement des problèmes des enfants, mais aussi par l'accès à des services plus appropriés. Dans le premier cas, la plupart des enfants sont devenus moins perturbés et plus disciplinés dans leurs habitudes. En conséquence, la vie familiale est devenue plus routinière et moins stressante, obligeant moins les parents à chercher du soutien. Toutefois, tous les enfants sont toujours significativement atteints et les espoirs initiaux des parents en une amélioration substantielle ne sont guère tenables. Dans l'étude de Veyssières et al. [34] déjà citée, les parents mentionnent, dans les difficultés actuelles, la force physique de leur enfant devenue adolescent et la fréquence d'un comportement agressif (autodirigé ou hétérodirigé, oppositionnel), ou en tout cas plus manifeste (cris, gestes...); des troubles du sommeil (apparition d'une altération du rythme circadien). Certains troubles des conduites alimentaires sont aussi rapportés, ainsi que des troubles du contrôle sphinctérien ; enfin, avec la puberté, des comportements sexuels inadaptés viennent déstabiliser le parent. Le faire face actuel des parents change corrélativement et se focalise sur l'autonomisation maximale de l'enfant devenu adolescent (avec en creux l'inquiétude quant à son devenir après leur propre disparition).

\subsection{Sur le lien entre la sévérité et le «stress»}

D'autres études confirment le lien entre le degré de gravité du trouble autistique et le stress parental [15,22]. Il apparaît évident que moins le trouble est sévère (pas de comorbidité, peu de comportements inadaptés...), moins la mère se montre pessimiste et plus la relation est vécue de façon plus positive. La stratégie de la «régulation émotionnelle » (contrôle des affects) semble modérer le lien entre le pessimisme parental et la symptomatologie autistique. De même que la stratégie de «distraction » modère le lien entre le stress et les symptômes de l'autisme [18]. Quand les symptômes autistiques sont moins sévères, l'engagement maternel (recherche de soutien social, rôle actif, organisation, engagement affectif) n'est pas relié au bien-être maternel. En revanche, quand les symptômes sont plus sévères, l'engagement exerce un effet positif sur le bien-être maternel. Quand les comportements inadaptés sont importants, les mères qui rapportent un engagement fort montrent un niveau de bien-être plus élevé que 
les mères dont l'engagement est moins important [5]. On peut penser que plus la situation est stressante et plus nous éprouvons un bien-être légitimé quand on réussit à la surmonter par ses exigences morales (tout faire pour que l'enfant aille mieux) ou le refus de la facilité (et donc du désengagement). Le langage autistique et les troubles cognitifs ont été jugés comme étant les plus sévères et les plus stressants par les mères et les pères de 20 enfants autistes [4]. Les parents d'enfants plus âgés ont toutefois tendance à diminuer le degré de sévérité, et les pères rapportent plus volontiers un haut degré de stress en continu. Selon Allik et al. [2], la santé maternelle (et non paternelle) serait davantage liée à l'intensité des problèmes de comportements de l'enfant tels que l'hyperactivité et les troubles des conduites. Cette étude suggère que la coexistence de problèmes comportementaux pourrait être plus stressante pour les parents que la sévérité du trouble autistique en lui-même. Le stress maternel pourrait être davantage corrélé aux problèmes de comportements de l'enfant qu'aux symptômes autistiques [14]. Il semble donc que les « problèmes de conduite » ou de discipline, ajoutés au manque de comportements sociaux des enfants soient des facteurs prédictifs forts du stress [17,23].

\section{Mère vs père}

Nous avons pu constater que la parole paternelle semble difficile : ce sont les mères qui répondent en majorité aux études. Le sex-ratio, très largement en faveur des femmes, est une donnée courante dans toute enquête. Il s'explique en partie par le fait que ce sont principalement les mères qui gèrent la vie quotidienne, voire élèvent leur enfant seule. Quelques études sur la qualité de vie des parents font effectivement état d'une différenciation sexuelle. Celle de Pelchat et al. [24] fait une synthèse très documentée des différences entre les pères et les mères d'enfants souffrant d'un problème de santé (cancer, déficience, trisomie $21 \ldots$... Concernant plus spécifiquement les TED, des différences apparaissent tant au niveau des effets affectifs que des stratégies déployées.

Selon Hastings et al. [14], les mères $(n=48)$ témoigneraient davantage d'état dépressif, mais aussi de perceptions positives (de leurs enfants de 37 mois en moyenne), que les pères $(n=41)$. Le stress paternel est par ailleurs davantage corrélé à l'état dépressif de la mère qu'à la situation de l'enfant. Les mères présenteraient également une santé physique plus faible que les pères [2]. Le symptôme de l'enfant considéré comme le plus gênant diffère également selon que l'on questionne les pères ou les mères [13]. Si les pères citent massivement les difficultés de communication verbale $(81 \%)$, les mères ont des réponses plus diversifiées : les troubles du comportement arrivent en première position (43,75\%), suivis par les difficultés de communication verbale $(25 \%)$, et l'absence de propreté $(18,75 \%)$. Cette 
différence s'explique par le fait que ce sont les mères qui consacrent plus d'heures à leur enfant que les pères. Gray [11] a interviewé 32 mères et 21 pères (australiens) d'enfants avec un autisme de haut niveau ou Asperger, âgés de 12 ans en moyenne (de 5 à 26 ans). Contrairement à leurs épouses, les pères estiment que la situation de leur enfant n'a pas d'effet personnel direct sur eux. Ils mettent plutôt en avant le poids sur leur famille (le stress de leurs épouses), et en particulier sur leur mariage. Cette conception est liée là aussi au rôle du père dans l'économie familiale : le père travaille et la mère s'occupe au quotidien de l'enfant. Ils reconnaissent d'ailleurs un engagement maternel plus grand. Les pères se considèrent comme des personnes ressources, ou de soutien, pour leur épouse et leur enfant. Les effets ressentis par les mères sont plus directs sur le plan émotionnel et sur le plan de la carrière professionnelle (la moitié seulement de l'échantillon ont un emploi, la plupart du temps à mi-temps). Dans la plupart des cas, les pères n'ont accompagné qu'occasionnellement les mères aux différents rendez-vous avec différents professionnels. Ces dernières se sentent fortement responsables, vis-à-vis de la famille et à l'extérieur (notamment l'école), du comportement de leur enfant. Connaissant bien le comportement de leur enfant, la stratégie parentale la plus employée face aux problèmes présentés par l'enfant est d'anticiper les difficultés et de programmer des réponses appropriées quand elles apparaissent. Mais quand les difficultés ne peuvent être anticipées, il s'agit alors de les prendre comme elles viennent, de vivre au jour le jour sans se remémorer ce qui est arrivé hier ni anticiper ce qui pourrait arriver. Parmi les pères, la stratégie la plus utilisée est de tenir l'enfant occupé (lui faire faire des choses). Pour les mères, il s'agit surtout de travailler avec leur enfant en participant à son régime thérapeutique ou éducatif, mais aussi de séparer l'enfant autiste de sa fratrie (pour l'épargner). Concernant la régulation émotionnelle, les pères déclarent surtout essayer de réprimer au maximum leurs sentiments. Mais l'émotion est parfois plus forte et c'est la colère qui constitue la principale réponse à un problème. Les mères tentent au contraire de laisser libre cours à leurs sentiments. Les émotions exprimées sont plus variées que celles des pères et associent la tristesse et le reproche à la colère. Contrairement aux pères, les mères disent aussi chercher à en parler à des amis ou à la famille ou, mieux, à d'autres mères ayant un enfant aussi handicapé. Interrogées sur la stratégie qui facilite le mieux le «coping », les mères citent d'ailleurs en premier le soutien (amis, famille, époux). La religion est aussi souvent évoquée. Les pères diffèrent par des stratégies beaucoup moins diversifiées et aucune de spécifique, à part de prendre la situation avec philosophie, de la relativiser.

Il s'avère que la nature du handicap, son degré de sévérité ou l'âge de la personne autiste (adulte/adolescent/enfant) ne sont pas les seuls critères qui influencent la façon dont le 
parent réagit et s'adapte à la situation. Le soutien du conjoint, tel qu'il est vécu, et du réseau social (famille, amis, voisins) y contribue aussi. Cela montre qu'il n'y a rien d'individuel dans le vécu de l'autisme, mais qu'il s'inscrit au contraire dans un faisceau de relations. Il reste que le soutien affectif en provenance du conjoint semble souvent faire défaut [29], et que les représentations négatives de la parentalité d'un enfant autiste affectent aussi la parentalité de la fratrie ayant un développement typique [19].

\section{Quelques écueils de l'évaluation psychosociale}

Retenons d'abord que les recherches citées dans ce travail, pour la plupart anglosaxonnes, sont difficilement comparables, tant les variables (notamment la tranche d'âge) et les méthodes employées sont disparates. Leurs limites tiennent aussi à l'emploi majoritaire d'une méthodologie statistique qui consiste à mesurer quantitativement le «stress » parental et/ou les «stratégies », sans en définir précisément les termes. La plupart ne viennent en fait que confirmer une intuition commune, à savoir par exemple [2] que les parents d'enfants handicapés sont plus «stressés », ont une santé plus fragile que les parents d'enfants au développement typique. Par ailleurs, les questionnaires (fermés) imposent nécessairement une échelle de mesure du «stress » ou de la qualité de vie, qui ne correspond pas nécessairement à celles mises en œuvre par les parents eux-mêmes. Cela signifie qu'il peut y avoir un décalage entre le questionnement professionnel et les inquiétudes parentales [26]. Enfin, si le trouble autistique affecte moralement les parents, on peut aussi raisonnablement penser que le stress parental affecte peu ou prou en retour l'enfant autiste. Cet impact éventuel est difficile à évaluer, mais ne peut être occulté pour autant.

\section{Conclusion}

Si l'analyse de l'épreuve parentale se justifie évidemment en elle-même, nous avons voulu mettre en avant dans ce travail le fait qu'elle puisse aussi éclairer sous un autre jour le syndrome autistique lui-même, et participer ainsi de son diagnostic. L'épreuve endurée dessine en creux le syndrome autistique dans toute sa complexité (l'isolement, l'absence d'intimité). Les études montrent que les parents ressentent plus douloureusement le trouble autistique que d'autres types de pathologies. Mais cette épreuve n'est jamais totalement négative. Cela signifie que les parents ne se contentent pas de subir le trouble de leurs enfants, mais cherchent activement à le positiver et le prendre en charge. Nous retenons que les effets 
positifs retenus sont souvent liés au fait d'avoir fait reculer les limites de l'insupportable, d'avoir surmonté de multiples épreuves grâce à un certain nombre de stratégies de «faire face ». La satisfaction semble se mesurer ainsi à la difficulté ou au défi lancé par l'autisme, trouble méconnu parce que rare. Mais cette attitude combative court peut-être le risque éventuel, partagé par les professionnels, d'un effondrement plus ou moins ponctuel, mais aussi le risque d'un masquage (déni) de son impuissance. Notre revue de la littérature montre aussi que le syndrome autistique de l'enfant n'affecte pas uniquement le parent mais réaménage l'ensemble de ses relations (conjugales, amicales, professionnelles). Le trouble autistique n'a rien d'individuel. Enfin, il faut tenir compte des différences entre les mères et les pères, qui apparaissent tant au niveau des effets affectifs que des stratégies déployées.

\section{Conflit d'intérêt : aucun}

\section{Références}

[1] Abbeduto L, Seltzer MM, Shattuck P, Krauss MW, Orsmond G, Murphy M. Psychological Well-Being and Coping in Mothers of Youths With Autism, Down Syndrome, or Fragile X Syndrome. American Journal On Mental Retardation 2004;109:237-54.

[2] Allik H, Larsson JO, Smedge H. Health-related quality of life in parents of school-age children with Asperger Syndrome or High-Functioning Autism. Health and Quality of Life Outcomes 2006. Available from: http://www.hqlo.com/content/4/1/1.

[3] Barron J, Barron S. Moi, l'enfant autiste. Paris: J'ai lu; 1995.

[4] Bebko J, Konstantareas MM, Springer J. Parent and professional evaluations of stress associated with characteristics of autism. Journal of Autism and Developmental Disabilities 1987;17:565-76.

[5] Benson PR. Coping, distress, and well-being in mothers of children with autism. Research in Autism Spectrum Disorders 2009; doi:10.1016/j.rasd.2009.09.008.

[6] Cappe N, Bobet R, Adrien JL. Psychiatrie sociale et problèmes d'assistance qualité de vie et processus d'adaptation des familles d'un enfant ayant un trouble autistique ou un syndrome d'asperger. La Psychiatrie de l'enfant 2009;1:201-46.

[7] Dale E, Knott F. Mothers' attributions following their child's diagnosis of autistic spectrum disorder. Exploring links with maternal levels of stress, depression and expectations about their child's future. Autism 2006;10:463-79.

[8] De Clercq H. Dis maman, c'est un homme ou un animal ? À propos de l'autisme. Mougins: Autisme France Diffusion; 2002. 
[9] Fleischmann A. Narratives Published on the Internet by Parents of Children With Autism What Do They Reveal and Why Is It Important? Focus on Autism and Other Developmental Disabilities 2004;19:35-43.

[10] Gray DE. Coping over time: The parents of children with autism. Journal of Intellectual Disability Research 2006;50:970-6.

[11] Gray DE. Gender and coping: the parents of children with high functioning autism. Social Science \& Medicine 2003;56:631-42.

[12] Green SE. We're tired, not sad: Benefits and burdens of mothering a child with a disability. Social Science \& Medicine 2007;64:150-63.

[13] Hahaut V, Castagna M, Vervier JF. Autisme et qualité de vie des familles. Louvain Med 2002;121:20-30.

[14] Hastings RP, Kovshoff H, Ward NJ, Espinosa FD, Brown T, Remington B. Systems analysis of stress and positive perceptions in mothers and fathers of pre-school children with autism. Journal of Autism and Developmental Disorders 2005;35:635-44.

[15] Ingersoll B, Hambrick DZ. The relationship between the broader autism phenotype, child severity, and stress and depression in parents of children with autism spectrum disorders. Research in Autism Spectrum Disorders 2010; doi:10.1016/j.rasd.2010.04.017.

[16] Kuhn JC, Carter AS. Maternal Self-Efficacy and Associated Parenting Cognitions Among Mothers of Children With Autism. American Journal of Orthopsychiatry 2006;76:564-75.

[17] Lecavalier L, Leone S, Wiltz J. The impact of behaviour problems on caregiver stress in young people with autism spectrum disorders. Journal of Intellectual Disability Research 2006;50:172-83.

[18] Lyons AM, Leon SC, Roecker Phelps CE, Dunleavy AM. The Impact of Child Symptom Severity on Stress Among Parents of Children with ASD: The Moderating Role of Coping Styles. Journal of Child and Family Studies 2009; doi : 10.1007/s10826-009-9323-5.

[19] Meirsschaut M, Roeyers H, Warreyn P. Parenting in families with a child with autism spectrum disorder and a typically developing child: Mother's experiences and cognitions. Research in Autism Spectrum Disorders 2010;4:661-9.

[20] Mugno D, Ruta L, d'Arrigo VG, Mazzone L. Impairment of quality of life in parents of children and adolescents with pervasive developmental disorder. Health and Quality of Life Outcomes 2007. Available from: http://www.hqlo.com/content/5/1/22. 
[21] Myers BJ, Mackintosh VH, Goin-Kochel RP. My greatest joy and my greatest heart ache: Parents' own words on how having a child in the autism spectrum has affected their lives and their families' lives. Research in Autism Spectrum Disorders 2009;3:670-84.

[22] Orsmond GI, Seltzer MM, Greenberg JS, Krauss MW. Mother-Child Relationship Quality Among Adolescents and Adults With Autism. American Journal on Mental Retardation 2006;111:121-37.

[23] Osborne LA, Reed P. Stress and self-perceived parenting behaviors of parents of children with autistic spectrum conditions. Research in Autism Spectrum Disorders 2010;4:405-14.

[24] Pelchat D, Lefebvre H, Levert MJ. L'expérience des pères et mères ayant un enfant atteint d'un problème de santé : état actuel des connaissances. Enfances, Familles, Générations 2005;3. http://id.erudit.org.scdbases.uhb.fr/iderudit/012536ar.

[25] Perrages P. Aux frontières de l'autisme, paroles de père. Colmar: Do Bentzinger éd.; 2003.

[26] Philip C. Autisme et parentalité. Paris: Dunod; 2009.

[27] Pottie CG, Ingram KM. Daily Stress, Coping, and Well-Being in Parents of Children With Autism: A Multilevel Modeling Approach. Journal of Family Psychology 2008;22:85564.

[28] Quentel JC. Le problème de la culpabilité chez les parents d'enfants handicapés. Neuropsychiatrie de l'enfance et de l'adolescence 1997;45:487-95.

[29] Roeyers H, Cloetens C. Les pères et les mères d'un enfant handicapé : leur adaptation à la situation et leur sentiment d'être aidés. Une comparaison entre des familles ayant un enfant souffrant d'autisme, un enfant avec un handicap mental ou un enfant dont le développement est normal. Dialogues 1995;2:3-10.

[30] Samios C, Pakenham KI, Sofronoff K. The nature of sense making in parenting a child with Asperger syndrome. Research in Autism Spectrum Disorders 2008;2:516-32.

[31] Schieve LA, Blumberg SJ, Rice C, Visser SN, Boyle C. The Relationship Between Autism and Parenting Stress. Pediatrics 2007;119:114-21.

[32] Seltzer MM, Kraus MW, Orsmond GI. Families of Adolescents and Adults with Autism: Uncharted Territory. International Review of Research in Mental Retardation 2001;23:26794.

[33] Sénéchal C, des Rivières-Pigeon C. Impact de l'autisme sur la vie des parents. Santé mentale au Québec 2009;34:245-60. 
[34] Veyssière A, Bernadoy C, Dagot L, Goussé V. Étude qualitative de l'évolution des stratégies de faire face chez les parents d'adolescents atteints de troubles autistiques. Pratiques psychologiques 2009; doi:10.1016/j.prps.2009.04.005. 